\title{
Vulnerabilidade programática para insegurança alimentar de crianças expostas ao HIV: revisão integrativa
}

\author{
Programmatic vulnerability to food insecurity of HIV-exposed infants: an integrative \\ review
}

\section{Vulnerabilidad programática para inseguridad alimentaria de niños expuestos al VIH: una revisión integradora}

Recebido: $14 / 08 / 2019$

Aprovado: $29 / 12 / 2020$

Marília Alessandra Bick ${ }^{1}$

Publicado: 17/02/2020

\section{Cristiane Cardoso de Paula²}

Esta é uma revisão integrativa, realizada nas bases LILACS, IBECS, PubMed e Scopus, em setembro de 2019, com o objetivo de analisar as evidências da literatura científica de fatores que influenciam na vulnerabilidade programática para insegurança alimentar de crianças expostas ao HIV. Foram incluídos 22 artigos primários publicados em português, inglês ou espanhol, sem corte temporal foram considerados, que apesar disto, apareceram a partir do ano de 2004 até 2019. A análise das evidências possibilitou a integração dos resultados desses artigos em três fatores que aumentam ou diminuem a vulnerabilidade: opções de alimentação, conhecimentos de atitudes e práticas dos profissionais e estrutura dos serviços. Como fatores de risco: burocracia para acesso gratuito à fórmula láctea, falha nas orientações para boas práticas de alimentação, estigma, mudanças nas diretrizes, acesso a diferentes serviços e insumos insuficientes. Concluímos que há necessidade de qualificar as orientações de opções de alimentação para as famílias dessas crianças, articulando as diretrizes das políticas públicas com as práticas dos profissionais de saúde e a estrutura dos serviços.

Descritores: HIV; Transmissão vertical de doença infecciosa; Nutrição do lactente; Políticas públicas de saúde.

This is an integrative review, carried out in the LILACS, IBECS, PubMed and Scopus databases, in September 2019, with the object of analyzing the evidence from scientific literature of factors that influence the programmatic vulnerability to food insecurity of HIV-exposed infants. Twenty-two primary articles published in Portuguese, English or Spanish were included. The analysis of the evidence made it possible to integrate the results of the articles into three factors that increase or decrease vulnerability: feeding options, knowledge of attitudes and practices of professionals and service structure. As risk factors: bureaucracy for free access to formula milk, failure in guidance for good feeding practices, stigma, changes in guidelines, access to different services and insufficient supplies. We conclude that there is a need to qualify the guidance for feeding options for the families of these infants, articulating the guidelines of public policies with the practices of health professionals and the structure of services.

Descriptors: HIV; Infectious disease transmission, Vertical; Infant nutrition; Public health policy.

Esta es una revisión integradora, realizada en las bases de datos LILACS, IBECS, PubMed y Scopus, en septiembre de 2019, con el objetivo de analizar las evidencias de la literatura científica de los factores que influyen en la vulnerabilidad programática para la inseguridad alimentaria de niños expuestos al VIH. Se incluyeron 22 artículos primarios publicados en portugués, inglés o español, sin recorte temporal fueron considerados, que a pesar de esto, aparecieron a partir del año 2004 hasta 2019. El análisis de las evidencias posibilitó la integración de los resultados de los artículos en tres factores que aumentan o diminuyen la vulnerabilidad: opciones de alimentación, conocimientos de actitudes y prácticas de profesionales y estructura de servicios. Como factores de riesgo: burocracia para el libre acceso a la fórmula láctea, falla en las orientaciones para buenas prácticas de alimentación, estigma, cambios en las directrices, acceso a diferentes servicios y insumos insuficientes. Concluimos que hay necesidad de calificar las orientaciones de opciones de alimentación para las familias de estos niños, articulando las directrices de las políticas públicas con las prácticas de los profesionales de la salud y la estructura de los servicios.

Descriptores: VIH; Transmisión vertical de enfermedad infecciosa; Nutrición del lactante; Políticas públicas de salud.

\footnotetext{
1. Nutricionista. Especialista em Gestão Pública em Saúde. Especialista em Alimentação Escolar. Mestre em Enfermagem. Doutoranda do Programa de Pós-Graduação em Enfermagem (PPGENF) da Universidade Federal de Santa Maria (UFSM), Santa Maria, RS, Brasil. 0RCID: 0000-0002-8744-7790 E-mail: mariliabick@gmail.com

2. Enfermeira. Especialista em Enfermagem Pediátrica. Especialista em Saúde do Adolescente. Mestre e Doutora em Enfermagem. Bolsista de Produtiva em Pesquisa do CNPQ. Professora Associada do PPGENF da UFSM, Santa Maria, Rio Grande do Sul, Brasil. ORCID: 0000-0003-4122-5161 E-mail: cristiane.paula@ufsm.br
} 


\section{INTRODUÇÃO}

$\mathbf{E}$ m âmbito mundial, a partir do ano 2010, as diretrizes da Organização Mundial da Saúde (OMS) orientam a promoção e apoio do aleitamento materno exclusivo para todas as crianças nascidas em regiões com alta ocorrência de mortalidade infantil por doenças diarreicas, pneumonias e desnutrição ${ }^{1}$. Nesses cenários, as diretrizes mantém a recomendação de prática do aleitamento materno mesmo na vigência da infecção pelo Vírus da Imunodeficiência Humana (HIV), entretanto, destaca a necessidade de adesão da mãe à Terapia Antirretroviral (TARV) e à manutenção do acompanhamento clínico e laboratorial da mãe e da criança ${ }^{1}$.

No ano de 2012, a atualização dessas diretrizes indicou a necessidade de decisão de cada país, considerando os índices locais de HIV, as condições gerais de saúde materna e infantil, e a capacidade dos sistemas de saúde em suprir as demandas da população para promover e apoiar práticas adequadas de alimentação e ampliar as ações de redução da transmissão vertical do HIV$^{2}$. As evidências apontam que, em regiões com alto índice de insegurança alimentar, a prática do aleitamento materno reduz a mortalidade e melhora o estado nutricional das crianças no primeiro ano de vida ${ }^{3,4}$. Contudo, efeitos adversos da exposição aos medicamentos antirretrovirais em longo prazo demandam atenção à saúde das crianças amamentadas 5 .

Os substitutos do leite materno devem ser considerados como opção para alimentar crianças verticalmente expostas ao HIV, quando forem aceitáveis, viáveis, acessíveis, sustentáveis e seguros ${ }^{6}$. Para isso, torna-se imprescindível que a família disponha de água potável, saneamento básico no domicílio e condições financeiras para prover a fórmula láctea infantil em quantidade suficiente para o crescimento adequado da criança ${ }^{5,6}$; conhecimentos e habilidades para prepará-la em quantidade e qualidade adequada, e oferecê-la de maneira oportuna à idade, exclusivamente até o sexto mês. Além de ser uma prática aceitável e apoiada pelo restante da família, também demanda acesso aos serviços de saúde para acompanhamento do crescimento e desenvolvimento infantil ${ }^{5,6}$.

No Brasil, a transmissão vertical do HIV constitui a principal categoria de exposição em menores de treze anos de idade, e representa 93,1\% dos casos notificados ${ }^{7}$. As taxas de transmissão podem reduzir a níveis inferiores a $2 \%$, quando o diagnóstico precoce das gestantes é realizado e são aplicadas todas as medidas profiláticas preconizadas pelo Ministério da Saúde. Dentre elas, destaca-se a necessidade de adesão à TARV ainda no pré-natal, bem como o acompanhamento clínico em Serviço de Atenção Especializada (SAE), durante o parto com profilaxia até o momento do clampeamento do cordão umbilical, indicação de via de parto adequada e inibição da lactação; e, após o nascimento da criança, com profilaxia medicamentosa e substituição do aleitamento materno por fórmula láctea infantil8.

Apesar do leite materno ser o melhor alimento para uma criança, mulheres infectadas pelo HIV podem predispor seus filhos à vulnerabilidade da infecção ${ }^{9}$, visto que o aleitamento materno responde por 15 a 35\% dos casos de transmissão vertical ${ }^{1}$ e reduz o impacto positivo das intervenções de prevenção realizadas na gestação e no parto ${ }^{7}$. Para tal, a política brasileira que visa à eliminação da transmissão vertical do HIV, contraindica a prática do aleitamento materno ou aleitamento cruzado, e garante por meio da Lei 9313/96 o provimento gratuito da fórmula láctea infantil, pelo menos até o sexto mês de vida da criança ${ }^{10}$.

Nesse sentido, o conceito de vulnerabilidade presume a exposição ao HIV como uma causa que perpassa a individualidade, e associa-se a aspectos relacionados ao contexto de vida, da coletividade e da sociedade em que o indivíduo se insere ${ }^{11}$. A alimentação de crianças expostas ao HIV está submetida a decisões programáticas das políticas e serviços de saúde 5 .

Assim, a vulnerabilidade programática se configura como determinante de situações individuais e sociais, a partir de políticas, programas e serviços sociais, de saúde e educação que mobilizam recursos e ações para proteger os indivíduos de determinado agravo ${ }^{11}$. Portanto, o objetivo deste estudo foi analisar as evidências da literatura científica de fatores 
que influenciam na vulnerabilidade programática para insegurança alimentar de crianças expostas ao HIV.

\section{MÉTODO}

Estudo de revisão integrativa, construído a partir da questão: “Quais os fatores que influenciam na vulnerabilidade programática para insegurança alimentar de crianças verticalmente expostas ao HIV?". Para reunir e sintetizar os resultados de pesquisas primárias, foi estabelecido o objetivo desta revisão integrativa, definidos os critérios de inclusão e exclusão, extraídas as informações dos artigos selecionados e construída a análise e discussão dos resultados para apresentação das evidências ${ }^{12}$.

A busca das publicações foi realizada nas bases de dados eletrônicas Publisher Medline (PUBMED), e Elsevier SciVerse Scopus (SCOPUS), e a partir da Biblioteca Virtual em Saúde (BVS) para acessar a base Literatura Latino-Americana e do Caribe em Ciências da Saúde (LILACS) e o Índice Bibliográfico Español en Ciencias de la Salud (IBECS), em setembro de 2019.

A estratégia utilizada nas bases PubMed e Scopus foi: [HIV OR "Acquired immunodeficiency syndrome"] AND ["infant nutritional physiological phenomena" OR "feeding practices" OR "bottle feeding" OR "infant formula"] como Mesh Terms. A busca na BVS/LILACS e IBECS foi realizada, por meio da estratégia: [HIV OR AIDS OR "Síndrome da imunodeficiência adquirida" OR "vírus da imunodeficiência humana" OR "Soropositividade para HIV"] AND [neonato OR criança OR pediatria OR "materno-infantil" OR "materno-fetal"] AND [nutrição OR alimentação OR "alimentação artificial" OR cuidadores OR cuidado OR "cuidado infantil"], selecionando no primeiro campo de busca os descritores de assunto, e nos demais campos, os títulos, resumos e assunto. Na biblioteca virtual, foi aplicado o filtro LILACS e IBECS.

Foram consideradas todas as produções indexadas nas bases de dados, até a data de busca e não considerando-se recorte temporal das produções. Para seleção das publicações, foram considerados como critérios de inclusão: artigos de pesquisa primária, publicados nos idiomas inglês, português ou espanhol. Aqueles duplicados nas bases de dados foram considerados apenas uma vez.

Para garantir a confiabilidade da seleção das produções, dois revisores realizaram a leitura e extração dos dados, de maneira independente, e nos casos de divergência de seleção, um terceiro pesquisador (orientador) realizou a leitura para seleção ou exclusão da produção.

Foi realizada uma avaliação crítica dos estudos a partir do sistema de classificação de força da evidência ${ }^{14}$. Nessa avaliação, a força da evidência considera a questão clínica de pesquisa do estudo primário, e classifica três tipos de hierarquia: estudos de tratamento/intervenção; estudos de prognóstico/etiologia e estudos de significado. 0 acesso aos artigos foi realizado na própria base de dados, e quando indisponíveis, a busca foi realizada no Portal da Coordenação de Aperfeiçoamento de Pessoal (CAPES), e também nos endereços eletrônicos dos periódicos.

Depois da leitura dos artigos selecionados, foi preenchido um instrumento de extração dos dados contendo a caracterização a partir do código de identificação da produção, o objetivo, o cenário de pesquisa e a população estudada. A extração dos resultados (evidências) foi desenvolvida considerando as situações individuais e sociais das famílias de crianças expostas ao HIV, a partir da maneira que políticas, programas e serviços mobilizam recursos e ações para protegê-las da insegurança alimentar.

Foram extraídas informações acerca das práticas de aleitamento materno; do acesso à fórmula láctea infantil; dos conhecimentos, atitudes e práticas dos profissionais de saúde; da estrutura dos serviços de saúde; das dificuldades e facilidades encontradas para alimentar as crianças verticalmente expostas ao HIV. 


\section{RESULTADOS}

A busca totalizou 1.175 produções para análise, das quais 300 da BVS/LILACS e IBECS, 313 da Scopus e 562 da PubMed. 0 corpus da pesquisa foi composto por 22 artigos de pesquisa primária (Figura 1).

Figura 1. Fluxograma de seleção dos artigos nas bases LILACS, IBECS, PubMed e Scopus, Santa Maria, RS, 2019.
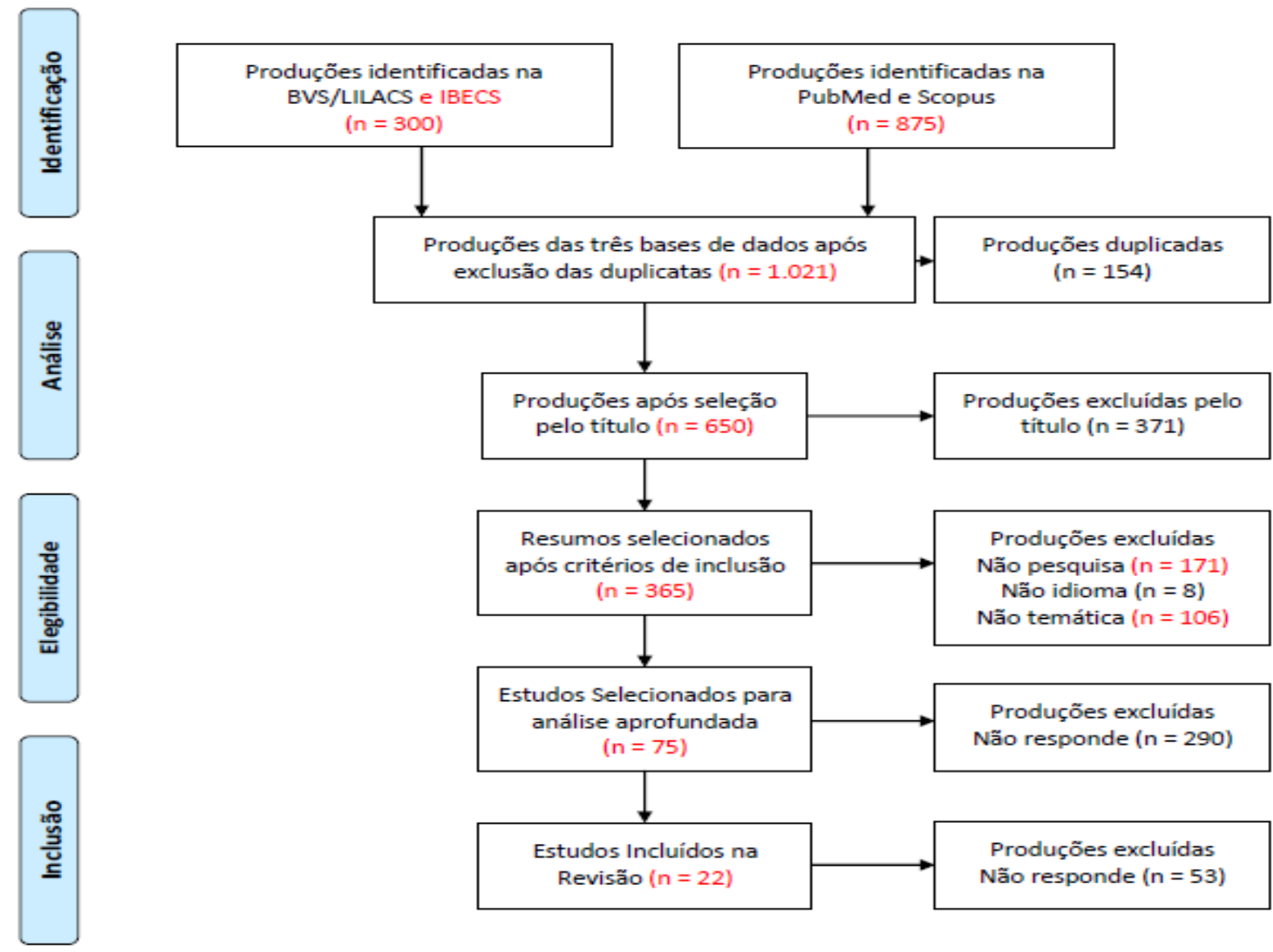

Fonte: Adaptado de MOHER et al. (2009) ${ }^{13}$

Os estudos de evidência forte foram aqueles que apresentavam por objetivo conhecer o significado das práticas de alimentação oferecidas as crianças verticalmente expostas ao HIV (Quadro 1). E, apesar de não haver corte temporal, os estudos considerados se deram após o ano de 2004, com apenas quatro estudos brasileiros e 15 estudos exclusivamente africanos e dois com outros locais e incluso o continente africano.

Por sua vez, a análise das publicações $(n=22)$ possibilitou a identificação das situações de vulnerabilidade programática para insegurança alimentar de crianças expostas ao HIV. Essas situações referem-se ao modo como os serviços de saúde e demais instâncias sociais efetivam o cuidado à saúde das crianças no que tange à alimentação na vigência do HIV (Figura 2). 
Quadro 1. Artigos incluídos na revisão integrativa de vulnerabilidade programática para a insegurança alimentar e nutricional. Santa Maria, RS, 2019.

\begin{tabular}{|c|c|c|c|c|}
\hline REF & $\begin{array}{l}\text { Ano } \\
\text { País } \\
\end{array}$ & Objetivo & Delineamento & NE \\
\hline 15 & $\begin{array}{c}2004 \\
\text { Camarões }\end{array}$ & $\begin{array}{l}\text { Determinar a proporção de mães que escolheram } \\
\text { diferentes métodos de alimentação, determinar os } \\
\text { vários fatores que influenciam suas escolhas e as } \\
\text { relações desses fatores com suas respectivas escolhas. }\end{array}$ & $\begin{array}{l}\text { Estudo qualitativo. } \mathrm{P} \\
=108 \text { mães HIV } \\
\text { positivas. }\end{array}$ & $4 \dagger$ \\
\hline 16 & $\begin{array}{l}2006 \\
\text { África do } \\
\text { Sul }\end{array}$ & $\begin{array}{l}\text { Examinar as características das mulheres HIV } \\
\text { positivas e seus ambientes que contribuíram para o } \\
\text { sucesso na manutenção da amamentação exclusiva ou } \\
\text { da fórmula exclusiva. }\end{array}$ & $\begin{array}{l}\text { Entrevista } \\
\text { qualitativa } \\
\text { longitudinal. P = } 27 \\
\text { mulheres com } \\
\text { resultado positivo no } \\
\text { teste de HIV durante } \\
\text { o pré-natal. }\end{array}$ & $4 \dagger$ \\
\hline 17 & $\begin{array}{l}2006 \\
\text { África do } \\
\quad \text { Sul }\end{array}$ & $\begin{array}{lllll}\text { Explorar como a epidemia do vírus } & \text { da } \\
\text { imunodeficiência humana (HIV) afetou } & \text { as } \\
\text { experiências de alimentação infantil de mães } & \text { HIV } \\
\text { positivas na África do Sul. } & & & \end{array}$ & $\begin{array}{l}\text { Estudo de entrevista } \\
\text { qualitativa em uma } \\
\text { coorte prospectiva. } P \\
=40 \text { mulheres. }\end{array}$ & $2 \neq$ \\
\hline 18 & $\begin{array}{c}2007 \\
\text { Brasil }\end{array}$ & $\begin{array}{l}\text { Conhecer, sob a visão de mães HIV positiva, como se } \\
\text { dá o acesso à doação de fórmula infantil nas Unidades } \\
\text { de Saúde e a utilização de alimentos entre crianças de } \\
\text { zero a dois anos. }\end{array}$ & $\begin{array}{l}\text { Estudo descritivo e } \\
\text { exploratório, de } \\
\text { natureza qualitativa. } \\
\mathrm{P}=15 \text { mulheres } \\
\text { soropositivas. }\end{array}$ & $2 \neq$ \\
\hline 19 & $\begin{array}{c}2007 \\
\text { Brasil }\end{array}$ & $\begin{array}{l}\text { Avaliar como a política de prevenção de HIV, } \\
\text { particularmente o componente alimentação infantil, } \\
\text { está implantado em São Paulo e como podemos } \\
\text { corrigir os erros e melhorar a assistência prestada a } \\
\text { esse respeito. }\end{array}$ & $\begin{array}{l}\text { Estudo descritivo. P } \\
=118 \text { observações } \\
\text { estruturadas das } \\
\text { visitas das mães aos } \\
\text { profissionais de } \\
\text { saúde. }\end{array}$ & $4 \dagger$ \\
\hline 20 & $\begin{array}{l}2008 \\
\text { Botsuana, } \\
\text { Quênia, } \\
\text { Malawi e } \\
\text { Uganda } \\
\end{array}$ & $\begin{array}{l}\text { Avaliar os componentes de alimentação infantil nos } \\
\text { programas de prevenção da transmissão do HIV de } \\
\text { mãe para filho (PTV). }\end{array}$ & $\begin{array}{l}\text { Estudo descritivo } \\
\text { transversal. } \mathrm{P}=334 \\
\text { trabalhadores da } \\
\text { saúde envolvidos no } \\
\text { programa de PTV. }\end{array}$ & $4 \dagger$ \\
\hline 21 & $\begin{array}{l}2008 \\
\text { África do } \\
\text { Sul, } \\
\text { Namíbia e } \\
\text { Suazilândia }\end{array}$ & $\begin{array}{l}\text { Explorar as percepções de mães e conselheiros de } \\
\text { saúde acerca dos encontros de aconselhamento de } \\
\text { alimentação infantil. }\end{array}$ & $\begin{array}{l}\text { Pesquisa etnográfica. } \\
\mathrm{P}=248 \text { pessoas } \\
\text { envolvidas no } \\
\text { programa de PTV. }\end{array}$ & $2 \neq$ \\
\hline 22 & $\begin{array}{l}2009 \\
\text { África do } \\
\text { Sul }\end{array}$ & $\begin{array}{l}\text { Compreender os fatores facilitadores e desafiadores } \\
\text { que afetam as práticas de alimentação infantil em } \\
\text { comunidades com alta prevalência de HIV. }\end{array}$ & $\begin{array}{l}\text { Estudo qualitativo } \\
\text { com entrevistas e } \\
\text { observações } \\
\text { aprofundadas. } \mathrm{P}=11 \\
\text { Mães recrutadas em } \\
\text { uma clínica de HIV }\end{array}$ & $2 \neq$ \\
\hline 23 & $\begin{array}{c}2009 \\
\text { Burkina } \\
\text { Faso, } \\
\text { Camboja e } \\
\text { Camarões } \\
\end{array}$ & $\begin{array}{l}\text { Mostrar características gerais e características locais, } \\
\text { para fornecer um entendimento do aconselhamento e } \\
\text { escolha de alimentação infantil, a partir das relações } \\
\text { entre as mulheres HIV positivas e o sistema de saúde. }\end{array}$ & $\begin{array}{l}\text { Abordagem } \\
\text { qualitativa. P }=159 \\
\text { mães HIV }+ \text { em } \\
\text { programas de PTV. }\end{array}$ & $4 \dagger$ \\
\hline 24 & $\begin{array}{l}2010 \\
\text { Burkina } \\
\text { Faso }\end{array}$ & $\begin{array}{l}\text { Explorar a aceitabilidade e a viabilidade das duas } \\
\text { principais opções de alimentação infantil em um } \\
\text { contexto urbano em Burkina Faso. }\end{array}$ & $\begin{array}{lr}\text { Discussões } & \text { em } \\
\text { grupos focais. } \mathrm{P}=17 \\
\text { mães } & \text { que } \\
\text { amamentam } & \text { com } \\
\text { fórmula e } 19 & \text { com } \\
\text { leite materno. } & \\
\end{array}$ & $2 \neq$ \\
\hline 25 & $\begin{array}{c}2010 \\
\text { Zâmbia }\end{array}$ & $\begin{array}{l}\text { Investigar até que ponto as opções de alimentação } \\
\text { infantil das mulheres do CIGNIS refletiram as } \\
\text { recomendações, com base em dados socioeconômicos } \\
\text { coletados como parte do estudo. }\end{array}$ & $\begin{array}{l}\text { Pesquisa quanti- } \\
\text { qualitativa. } \mathrm{P}=811 \\
\text { mães de bebês. }\end{array}$ & $6^{*}$ \\
\hline
\end{tabular}




\begin{tabular}{|c|c|c|c|c|}
\hline 26 & $\begin{array}{l}2013 \\
\text { África do } \\
\quad \text { Sul }\end{array}$ & $\begin{array}{l}\text { Explorar as percepções e compreensões das famílias, } \\
\text { sobre a decisão política de eliminar progressivamente } \\
\text { a gratuidade da fórmula láctea no programa de PTV da } \\
\text { África do Sul. }\end{array}$ & $\begin{array}{l}\text { Estudo qualitativo } \\
\text { exploratório. P }=60 \\
\text { partipicantes: } 11 \\
\text { mães soropositivas, } \\
9 \quad \text { mães } \\
\text { soronegativas e seus } \\
\text { familiares. }\end{array}$ & $2 \neq$ \\
\hline 27 & $\begin{array}{l}2012 \\
\text { África do } \\
\quad \text { Sul }\end{array}$ & $\begin{array}{l}\text { Explorar as percepções e experiências das mães sobre } \\
\text { alimentação infantil, a partir de uma intervenção } \\
\text { comunitária de aconselhamento por pares que } \\
\text { promove a exclusividade da amamentação ou fórmula. }\end{array}$ & $\begin{array}{lr}\text { Estudo qualitativo } \\
\text { exploratório. } P=17 \\
\text { mães } \\
\text { participaram } \quad \text { da } \\
\text { intervenção } \\
\text { PROMISE-EBF. }\end{array}$ & $2 \neq$ \\
\hline 28 & $\begin{array}{l}2013 \\
\text { África do } \\
\quad \text { Sul }\end{array}$ & $\begin{array}{l}\text { Explorar as influências nas intenções e práticas de } \\
\text { alimentação infantil de mulheres vivendo com HIV na } \\
\text { África do Sul. }\end{array}$ & $\begin{array}{l}\text { Estudo de } \\
\text { mistos. P } \quad \text { étodos } \\
\text { gestantes e } 207 \\
\text { puérperas. }\end{array}$ & $4 \dagger$ \\
\hline 29 & $\begin{array}{c}2014 \\
\text { Brasil }\end{array}$ & $\begin{array}{l}\text { Conhecer a experiência de cuidadores de crianças } \\
\text { expostas verticalmente ao HIV. }\end{array}$ & $\begin{array}{l}\text { Pesquisa } \\
\text { exploratória, } \\
\text { descritiva } \\
\text { qualitativa. } P=12 \\
\text { mães e uma avó. }\end{array}$ & $2 \neq$ \\
\hline 30 & $\begin{array}{c}2016 \\
\text { Brasil }\end{array}$ & $\begin{array}{l}\text { Conhecer as dificuldades e facilidades da família para } \\
\text { cuidar a criança com HIV/Aids. }\end{array}$ & $\begin{array}{l}\text { Estudo descritivo, } \\
\text { exploratório } \\
\text { qualitativo. } P=15 \\
\text { familiares. }\end{array}$ & $4 \dagger$ \\
\hline 31 & $\begin{array}{l}2016 \\
\text { África do } \\
\quad \text { Sul }\end{array}$ & $\begin{array}{l}\text { Determinar o conhecimento, opiniões e práticas de } \\
\text { profissionais de saúde em maternidades de um } \\
\text { hospital regional de Bloemfontein, África do Sul, sobre } \\
\text { alimentação infantil no contexto do HIV. }\end{array}$ & $\begin{array}{l}\text { Desenho descritivo } \\
\text { do estudo } \\
\text { transversal. } \mathrm{P}=64 \\
\text { trabalhadores } \\
\text { saúde. }\end{array}$ & $2 \neq$ \\
\hline 32 & $\begin{array}{l}2017 \\
\text { África do } \\
\text { Sul } \\
\end{array}$ & $\begin{array}{l}\text { Explorar os fatores que influenciam a escolha da } \\
\text { alimentação infantil de mães HIV positivas em um } \\
\text { hospital periurbano em Tembisa, África do Sul. }\end{array}$ & $\begin{array}{l}\text { Estudo qualitativo } \\
\text { exploratório. } \mathrm{P}=30 \\
\text { mães HIV positivas. }\end{array}$ & $4 \dagger$ \\
\hline 33 & $\begin{array}{l}2017 \\
\text { Tailândia }\end{array}$ & $\begin{array}{l}\text { Explorar as experiências de amamentação entre } \\
\text { mulheres tailandesas vivendo com HIV no sul da } \\
\text { Tailândia. }\end{array}$ & $\begin{array}{l}\text { Entrevistas } \\
\text { semiestruturadas e } \\
\text { métodos de desenho. } \\
\mathrm{P}=30 \text { mulheres HIV } \\
\text { positivas. }\end{array}$ & $2 \neq$ \\
\hline 34 & $\begin{array}{c}2018 \\
\text { Quênia }\end{array}$ & $\begin{array}{l}\text { Identificar barreiras à alimentação ideal entre } \\
\text { crianças expostas ao HIV de } 0 \text { a } 5 \text { meses de idade que } \\
\text { frequentam um hospital missionário no condado de } \\
\text { Bomet, no Quênia. }\end{array}$ & $\begin{array}{l}\text { Estudo qualitativo } \\
\text { transversal. P = } 35 \\
\text { mães / cuidadores } \\
\text { de bebês expostos ao } \\
\text { HIV. }\end{array}$ & $4 \dagger$ \\
\hline 35 & $\begin{array}{l}2018 \\
\text { África do } \\
\quad \text { Sul }\end{array}$ & $\begin{array}{l}\text { Exploramos como os profissionais de saúde } \\
\text { experimentaram essa nova política em uma } \\
\text { comunidade endêmica de HIV em 2015-16, com } \\
\text { atenção ao seu conhecimento da política, práticas de } \\
\text { aconselhamento e observações de quaisquer } \\
\text { mudanças. }\end{array}$ & $\begin{array}{lr}\text { Estudo transversal } \\
\text { de métodos mistos. P } \\
=\quad 46 \quad \text { mães } \\
\text { participaram } & \text { de } \\
\text { entrevistas } & \text { em } \\
\text { profundidade. }\end{array}$ & $2 \ddagger$ \\
\hline 36 & $\begin{array}{l}2019 \\
\text { África do } \\
\quad \text { Sul }\end{array}$ & $\begin{array}{l}\text { O objetivo deste artigo é determinar a taxa de adoção } \\
\text { do aleitamento materno exclusivo nesta coorte, } \\
\text { examinar os determinantes das opções de } \\
\text { alimentação infantil de mulheres infectadas pelo HIV } \\
\text { e avaliar as razões subjacentes a essas escolhas. }\end{array}$ & $\begin{array}{l}\text { Estudo de métodos } \\
\text { mistos. P }=1662 \\
\text { mulheres } \\
\text { peripartum. }\end{array}$ & $4 \dagger$ \\
\hline
\end{tabular}

Legendas: REF: Referência; NE: Nível de evidência; *Tratamento/intervenção; †Prognóstico/etiologia; ‡Significado. 
Figura 2 - Fatores de vulnerabilidade programática para insegurança alimentar de crianças expostas ao HIV ( $\mathrm{n}=22)$, Brasil, 2019.

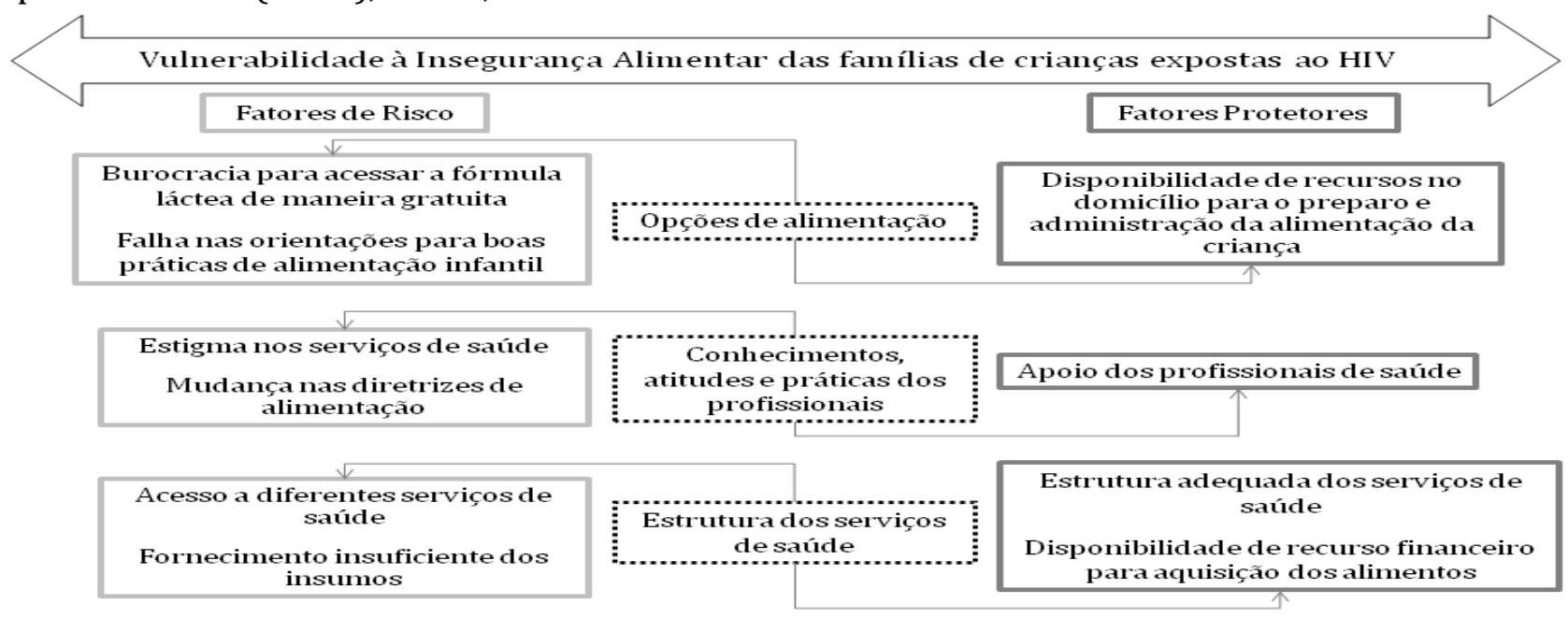

\section{DISCUSSÃO}

As dificuldades e facilidades para alimentar as crianças verticalmente expostas ao HIV foram evidenciadas pelas opções de alimentação, conhecimentos, atitudes e práticas dos profissionais de saúde e estrutura dos serviços de saúde.

$\mathrm{Na}$ escolha entre as opções de alimentação, os fatores que aumentam a vulnerabilidade para a insegurança alimentar foram: o fornecimento instável de FL gratuita ${ }^{16-17,26-29}$ e a falha no compromisso com orientações para boas práticas de alimentação quando os profissionais de saúde ofereciam poucas orientações acerca do custo de compra ${ }^{15-16,34}$, da necessidade de usar suprimento adequado de água ${ }^{34}$ e de manter o leite preparado refrigerado ${ }^{19}$. A confiança e o aconselhamento dos profissionais de saúde foram fundamentais para manutenção do aleitamento materno exclusivo (AME) ${ }^{15,36}$; o baixo nível socioeconômico inviabilizou a alimentação com fórmula láctea $(\mathrm{FL})^{22,25,33,36}$ e favoreceu o $\mathrm{AME}^{23}$; o suprimento gratuito e ininterrupto de fórmula, água potável encanada e energia elétrica no domicílio favoreceram a alimentação com FL ${ }^{16,24-25}$.

Os conhecimentos, atitudes e práticas dos profissionais de saúde aumentaram a vulnerabilidade quando houve preconceito e estigma, com revelação do diagnóstico a pessoas da comunidade18,23,30; imposição de não amamentação, sem considerar as condições das famílias 16,19,21,23,27,28,32,33,36; falta de orientações de introdução de alimentação complementar32.

Os profissionais consideraram que a orientação de preparo da FL era dever do nutricionista e parcialmente do enfermeiro ${ }^{19,34}$, sendo que estes últimos demonstraram maior habilidade para orientar as mães quanto à opção de alimentação ${ }^{31}$. As evidências da produção científica indicaram como fatores protetores da insegurança alimentar: o vínculo e o apoio psicossocial dos profissionais de saúde, desde o pré-natal até o acompanhamento em saúde das crianças expostas, foram positivos para as práticas adequadas de alimentação infantil 16,24,25,29.

Quanto à estrutura dos serviços de saúde, os fatores que aumentaram a vulnerabilidade foram: falta de suporte ou treinamento sobre as novas diretrizes de alimentação infantil e HIV20, ocasionando inconsistência nas orientações ${ }^{17,21,22,25,28,34,35}$; desorganização e falta de estrutura dos serviços de saúde para atender essa população ${ }^{18}$; estoque insuficiente ${ }^{16}$ e problemas no controle e distribuição da FL 19,20,26,35; assédio de indústrias de alimentos com oferta de amostras gratuitas de $\mathrm{FL}^{20}$; roubo e venda de FL por parte dos profissionais do serviço ${ }^{26}$; e a necessidade de acesso a diferentes serviços para retirada da FL foi descrita como estigmatizante 28 . Enquanto, os protetores da insegurança alimentar evidenciados foram: o fornecimento gratuito de FL demandou investimentos em infraestrutura para armazenamento 
e distribuição ${ }^{20}$; e o benefício governamental de complementação de renda, aconselhamento em grupos de apoio favoreceram a alimentação adequada das crianças ${ }^{23,30}$.

Os aspectos programáticos estão intimamente relacionados à vulnerabilidade para insegurança alimentar de crianças verticalmente expostas ao HIV. Os estudos evidenciaram que as famílias buscam evitar a transmissão vertical do HIV para os filhos. Assim, optam por um tipo de alimentação, seja pelo aleitamento materno seja pela alimentação com fórmula láctea infantil. Independente das condições sociais e econômicas, as famílias compreendem a necessidade de manter a exclusividade do tipo de alimentação escolhida e se esforçam para tal.

Indica-se a importância de orientações acerca da influência da opção de alimentação na prevenção da transmissão vertical do HIV e da influência das boas práticas de alimentação infantil na promoção da segurança alimentar. Essas orientações devem ser fornecidas pelos profissionais de saúde desde o pré-natal até o puerpério e puericultura.

Foram evidenciadas as situações que aumentam a vulnerabilidade à insegurança alimentar das famílias de crianças expostas ao HIV: burocracia para acessar a fórmula láctea de maneira gratuita; falha no compromisso com orientações para boas práticas de alimentação infantil; estigma nos serviços de saúde; mudanças nas diretrizes de alimentação; acesso a diferentes serviços de saúde; e fornecimento insuficiente dos insumos, especialmente disponibilização gratuita de substitutos do leite materno.

A burocracia para acessar a fórmula láctea de maneira gratuita, foi destacada como uma barreira para a opção pela alimentação de substituição 15,17,22,26,32. No Brasil, estudos mostraram a inconstância do fornecimento da fórmula láctea ${ }^{18,29}$. As falhas no suprimento de fórmula láctea pelo serviço de saúde ou de assistência social foi um fator determinante de vulnerabilidade para insegurança alimentar, pois o alto custo semanal da fórmula ocasionou a introdução precoce da alimentação complementar ${ }^{16,18,22,28,29,32,34}$. Destaca-se a ocorrência de violações ao Código Internacional de Substitutos do Leite Materno, relatada pelos profissionais de saúde, a partir da oferta maciça de amostras grátis de fórmula láctea infantil, provenientes das indústrias fabricantes ${ }^{17,22}$.

Sobre isso se infere que não é suficiente a garantia por lei do acesso gratuito à fórmula láctea infantil, que nacionalmente é estabelecida por no mínimo seis meses. É necessária a articulação entre aquilo que está preconizado na política pública e a organização dos serviços de saúde para distribuição da fórmula láctea de maneira efetiva. As falhas nessa articulação repercutem na prática alimentar em quantidade e frequência inadequada para idade da criança e introdução precoce de outros alimentos.

A falha no compromisso com orientações para boas práticas de alimentação infantil ${ }^{32}$ gera dúvida e medo de adoecimento dos filhos para aquelas famílias que optaram pela alimentação de substituição, em razão das dificuldades de preparo da fórmula ${ }^{23,24,27}$ ou alergias à fórmula láctea infantil ${ }^{28}$. Em um serviço de saúde, o critério adotado para a recomendação da prática alimentar para cada criança exposta era determinado pelo nível de carga viral materna. Quando indetectável, orientava-se o aleitamento materno exclusivo. A fórmula láctea era recomendada quando a carga viral materna estava elevada, entretanto os profissionais de saúde relataram incerteza quanto à efetividade da recomendação ${ }^{26}$.

As evidências mostram a ocorrência de orientações inadequadas e inconsistentes, indicando a necessidade de melhorar a qualidade do aconselhamento em saúde realizado nos serviços $15-17,19,20,27,29,33$. Muitas vezes, em um mesmo local, profissionais orientavam as mulheres de maneira contraditória $15,19,25,26,32$. Divergências e falta de clareza entre as orientações que reforçavam a possibilidade do aleitamento materno deixavam subentendidos os riscos para a transmissão do HIV15,19,20,22,25,36.

A respeito disso, destaca-se a necessidade de coerência entre as recomendações de alimentação da criança para profilaxia de transmissão vertical e as orientações fornecidas pelos profissionais nos serviços de saúde. Isso indica a necessidade de profissionais capacitados e permanentemente atualizados, ou seja, que desenvolvam orientações sustentadas nas 
evidências científicas, em linguagem acessível à compreensão da família e às condições de preparo e administração da fórmula láctea e alimentação no domicílio. Então, essa articulação tende a diminuir a vulnerabilidade para a insegurança alimentar das crianças, por meio de orientações adequadas, evitando dúvidas de prática alimentar e minimizando medos de transmissão vertical do HIV.

O estigma vivido pelas mulheres em um local que deveria acolhê-las foi fator determinante para a descontinuidade do acompanhamento clínico ${ }^{21,23}$ no serviço e a descontinuidade da exclusividade da opção de alimentação com fórmula láctea ${ }^{18}$.

Nesse caso é reconhecível a importância da capacitação dos profissionais para o desenvolvimento de uma prática ética, baseada em evidências científicas. Mais uma vez, o estigma da doença impacta negativamente na longitudinalidade da atenção em saúde e, consequentemente, em desfechos preveníveis como a transmissão vertical do HIV e a insegurança alimentar.

A mudança nas diretrizes de alimentação de crianças expostas ao HIV, possibilitando que uma mulher infectada pelo HIV realizasse o aleitamento materno, foi visto como surpreendente em algumas situações ${ }^{20,22,25,30}$.

Observou-se conexões entre os fatores de risco para a vulnerabilidade à insegurança alimentar das famílias de crianças expostas ao HIV por meio de diferentes situações até então discutidas e que reforçam a necessidade de profissionais capacitados e permanentemente atualizados.

A necessidade de acesso a diferentes serviços de saúde em curto período de tempo, para o acompanhamento clínico, obtenção da fórmula láctea infantil e dos medicamentos antirretrovirais foi destacada de maneira negativa pelas mulheres, em razão da desorganização, despreparo e falta de acolhimento dos serviços ${ }^{18,29-30}$. A necessidade de revelar o diagnóstico nesses serviços causou constrangimento, em razão da atitude preconceituosa e recriminadora assumida por alguns profissionais de saúde 18,21,31.

Destaca-se a conexão entre organização dos serviços e capacitação dos profissionais para uma prática ética baseada em evidência

Quanto ao fornecimento insuficiente de insumos, em locais em que não há fornecimento gratuito da fórmula láctea infantil, as mães que não possuíam condições financeiras de arcar com o custo dessa escolha de alimentação, não tinham outra opção, a não ser optar pela amamentação mista15,22-23,25-26,31-32,35.

Em virtude do exposto, entende-se que isso reforça o reconhecimento mundial da política pública brasileira de prevenção da transmissão do HIV e de tratamento da AIDS. E ainda, sustenta o motivo da recomendação de aleitamento materno para aqueles cenários que não possuam essa cobertura de atenção às pessoas vivendo com HIV. As evidências aqui referidas de alimentação mista decorrente de fornecimento insuficiente de insumos são majoritariamente provenientes de estudos em países do continente africano, o que contextualiza essa situação indica a necessidade de orientação adaptada às situações de cada família, para que mantenha a exclusividade do tipo de alimentação, nesse caso, o aleitamento materno.

Foram evidenciadas, também, situações que diminuem a vulnerabilidade à insegurança alimentar das famílias de crianças expostas ao HIV: a disponibilidade de recursos financeiros para aquisição dos alimentos e de recursos no domicílio para o preparo e administração da alimentação da criança; apoio dos profissionais de saúde desde o pré-natal até o puerpério e puericultura; e estrutura adequada dos serviços de saúde.

A disponibilidade de recursos financeiros para aquisição do alimento, além de eletricidade e utensílios domésticos necessários para o seu preparo e administração facilitou a opção de alimentar os filhos com fórmula láctea infantil15,16,24,26. As mães se mostraram autoconfiantes e aliviadas por oferecerem uma forma adequada de alimentação aos filhos, além de não representam um risco de transmissão do HIV15,24,28,31. Mais importante do que os 
conhecimentos acerca dos riscos de transmissão do vírus e o desejo de amamentar ou de não amamentar, as condições socioeconômicas e de moradia definiram as práticas adotadas $^{22,23,25,26,31,34}$. A hiperdiluição da fórmula para suprir as necessidades dos demais filhos ou aumentar seu período de duração aumentaram o risco de insegurança alimentar das crianças que já possuem um sistema imunológico deficitário18,31.

Acredita-se que as condições sociais vivenciadas pelas famílias são determinantes da efetivação das práticas de alimentação das crianças expostas ao HIV. Os recursos financeiros para aquisição dos alimentos e de recursos no domicílio para o preparo e administração da alimentação da criança precisam ser considerados pelos profissionais na orientação acerca da opção alimentar, da manutenção de sua exclusividade por tempo oportuno e na prática cotidiana de cuidados, a fim de promover a segurança alimentar.

0 apoio dos profissionais, desde o pré-natal, foi considerado fundamental para auxiliar na prevenção da transmissão vertical do HIV 23,28 e no enfrentamento dos desafios e pressões que a escolha alimentar ocasionou 16,32 , sendo que o apoio psicossocial foi fator fundamental ao promover a autonomia quanto à escolha alimentar realizada15,24,28.

Os profissionais mostraram abertura para permitir que a mulher realizasse a escolha da melhor opção de alimentação para seu filho, considerando as circunstâncias do domicílio e as restrições práticas de cada uma das opções ${ }^{16,25}$. Ainda reforçaram a importância do relacionamento de empatia e confiança que tiveram com os profissionais de saúde do serviço ${ }^{24,28,32}$. Os enfermeiros foram considerados como fundamentais para a atenção à saúde das mulheres no puerpério ${ }^{33}$. E os nutricionistas foram indicados pelos demais profissionais de saúde como detentores e responsáveis pela orientação de alimentação infantil, e todos os entrevistados apresentaram conhecimentos adequados acerca das recomendações ${ }^{26,27}$.

Em contraponto, alguns orientaram a prática alimentar que consideravam adequada, sem ponderar o desejo da mãe 16,19,25-27,30-31. Ainda, as mães relataram forte repreensão realizada pelos profissionais de saúde quando não cumpriam as recomendações indicadas de alimentação infantil 15,27,33. Sentimentos como esgotamento, estresse e frustração também foram demonstrados pelos profissionais de saúde nessa situação ${ }^{19}$.

Acrescenta-se que, o apoio dos profissionais se deu por meio do aporte de informações acerca dos riscos de transmissão do HIV que a alimentação mista proporciona. Isso repercutiu no sucesso da exclusividade da escolha alimentar e na capacidade de resistir às pressões da família pela introdução de outros alimentos e líquidos ${ }^{16,32}$. Para as mães que optaram pelo aleitamento materno exclusivo, foi fundamental o aporte de informações acerca dos seus benefícios, destacando o vínculo entre mãe e filho além do desenvolvimento de crianças nutricionalmente saudáveis ${ }^{16,20,25,26,31}$, mesmo diante da preocupação recorrente com a possibilidade de transmissão do HIV para a criança ${ }^{22-24}$.

Assim, infere-se que o apoio psicossocial dos profissionais, inclusive por meio do aporte de informações, promove autonomia para escolha da opção de alimentação e sua manutenção exclusiva por tempo recomendado para segurança alimentar da criança. Para tanto, é necessário conhecimento para que o profissional oriente de modo adequado e que a família confie e se sinta confiante para a prática alimentar.

A estrutura adequada dos serviços de saúde e o fornecimento suficiente dos insumos, dentre eles os antirretrovirais e a fórmula láctea infantil foram destacados por todas as mães como fatores protetores da prática alimentar escolhida. A disponibilização de fórmula láctea infantil pelo governo demanda além de investimentos na compra desse alimento, em uma infraestrutura e logística diferenciada para armazenamento e distribuição adequadas ${ }^{33}$.

Reforça-se a indicação de articulação entre aquilo que está preconizado na política pública e a organização dos serviços de saúde. As falhas nessa articulação repercutem negativamente na prevenção da transmissão vertical do HIV e na segurança alimentar.

Os recursos sociais disponibilizados de modo efetivo e democrático protegem as crianças expostas ao HIV de exposição a outros agravos como a insegurança alimentar. Assim, as ações 
programáticas precisam articular planejamento, recursos, capacidade, gestão e avaliação de modo permanente desde a esfera política, perpassando pelos serviços e culminando na atuação profissional de modo a promover ações junto às famílias, contextualizadas à situação geográfica, demográfica, socioeconômica, cultural e clínica que vivenciam.

\section{CONCLUSÃo}

As opções de alimentação, os conhecimentos, as atitudes e as práticas dos profissionais, e a estrutura dos serviços aumentam ou diminuem a vulnerabilidade programática para insegurança alimentar de crianças expostas ao HIV. Os fatores de risco evidenciados foram: burocracia para acesso gratuito à fórmula láctea, falha nas orientações para boas práticas de alimentação, estigma, mudanças nas diretrizes, acesso a diferentes serviços de saúde e fornecimento insuficiente de insumos. Os fatores protetores foram: a disponibilidade de recursos no domicílio para o preparo e administração da alimentação da criança; o apoio dos profissionais de saúde; a estrutura adequada dos serviços e a disponibilidade de recursos financeiros para aquisição dos alimentos.

Verificou-se a necessidade de qualificar as orientações de opções de alimentação para as famílias dessas crianças, articulando as diretrizes das políticas públicas com as práticas dos profissionais de saúde e a estrutura dos serviços.

Como possíveis generalizações, dentre as ações programáticas para a garantia da segurança alimentar e nutricional de crianças expostas ao HIV, aponta-se que é preciso investir em atualização dos profissionais e adequação dos serviços de saúde para atender apropriadamente as demandas desta população vulnerável. As orientações precisam ser adaptadas às situações de cada família. Nos contextos em que as mulheres infectadas pelo HIV são orientadas a não amamentar, como no Brasil, destaca-se a necessidade de fornecimento contínuo e ininterrupto da fórmula láctea infantil.

$\mathrm{Na}$ avaliação crítica dos artigos, aponta-se que o quantitativo de nível $\leq 4$ indica a necessidade do desenvolvimento de estudos experimentais, coorte, caso controle e revisões sistemáticas. Dentre os 22 artigos incluídos, 11 foram classificados com nível 2, considerado forte, sendo majoritariamente qualitativos, indicando a necessidade de investimento em estudos de intervenção, para que além de ampliar as evidências se fortaleça a tomada de decisões na prática de alimentação de crianças expostas ao HIV, de modo a minimizar a vulnerabilidade programática para a insegurança alimentar.

Esta revisão integrativa apresenta limitações que se referem à complexidade de analisar artigos que utilizaram diferentes abordagens metodológicas; além da pluralidade de cenários com diferentes recomendações de alimentação (aleitamento materno ou formula láctea) para crianças expostas ao HIV, mas por outro lado, aponta o cenário dos estudos na temática, de grande importância para ações políticas e sociais.

\section{REFERÊNCIAS}

1. World Health Organization. Guidelines on HIV and infant feeding: principles and recommendations for infant feeding in the context of HIV and a summary of evidence [Internet]. Geneva: WHO; 2010 [citado em 05 mar 2019]. 58p. Disponível em: https://www.who.int/maternal_child_adolescent/documents/9789241599535/en/

2. World Health Organization. HIV and infant feeding 2010: an updated framework for priority action [Internet]. Geneva: WHO; 2012 [citado em 05 mar 2019]. 8p. Disponível em: https://www.who.int/maternal_child_adolescent/documents/9241590777/en/

3. Victora CG, Bahl R, Barros AJD, França GVA, Horton S, Krasevec J, et al. Breastfeeding in the 21st century: epidemiology, mechanisms, and lifelong effect. Lancet [Internet]. 2016 [citado em 05 mar 2019]; 387(10017):475-90. DOI: https://doi.org/10.1016/S0140-6736(15)01024-7

4. Santos GM, Cavalcanti AL, Vianna RPT, Silva CCS. Aleitamento materno exclusivo e (in) segurança alimentar e nutricional. Rev Bras Ciênc Saúde [Internet]. 2016 [citado em 05 mar 2019]; 20(4):293- 
8.

Disponível

em:

https://pdfs.semanticscholar.org/8602/dc5e8fdcb12a7cc5b98613414ebb476cf4a2.pdf. $\quad$ DOI: https://doi.org/10.4034/RBCS.2016.20.04.05

5. World Health Organization. Guideline updates on HIV and infant feeding: the duration of breastfeeding, and support from health services to improve feeding practices among mothers living with HIV [Internet]. Geneva: WHO; 2016 [citado em 05 mar. 2019]. 68p. Disponível em: https://www.who.int/maternal_child_adolescent/documents/hiv-infant-feeding-2016/en/

6. Doherty T, Chopra M, Jackson D, Goga A, Colvin M, Persson LA. Effectiveness of the WHO/UNICEF guidelines on infant feeding for HIV-positive women: results from a prospective cohort study in South Africa. AIDS [Internet]. 2007 [citado em 05 mar 2019]; 21(13):1791-7. DOI: https://doi.org/10.1097/QAD.0b013e32827b1462

7. Ministério da Saúde (Brasil), Secretaria de Vigilância em Saúde, Departamento de DST, Aids e Hepatites Virais. Boletim Epidemiológico HIV-AIDS [Internet]. 2018 [citado em 05 mar 2019]; 49(53):5-72. Disponível em: http://www.aids.gov.br/pt-br/pub/2018/boletim-epidemiologicohivaids-2018

8. Ministério da Saúde (Brasil), Departamento de Vigilância, Prevenção e Controle das Infecções Sexualmente Transmissíveis, do HIV/Aids e das Hepatites Virais. Protocolo clínico e diretrizes terapêuticas para manejo da infecção pelo HIV em crianças e adolescentes [Internet]. Brasília, DF: Ministério da Saúde; 2018 [citado em 05 mar 2019]. 222p. Disponível em: http://www.aids.gov.br/pt-br/pub/2017/protocolo-clinico-e-diretrizes-terapeuticas-paramanejo-da-infeccao-pelo-hiv-em-criancas-e

9. Freitas JG, Cunha GH, Lemos LA, Barroso LMM, Galvão MTG. Alimentação de crianças nascidas expostas ao vírus da imunodeficiência humana. Texto \& Contexto Enferm. [Internet]. 2014 [citado em 05 mar 2019]; 23(3):617-5. Disponível em: http://www.scielo.br/pdf/tce/v23n3/pt_01040707-tce-23-03-00617.pdf. DOI: http://dx.doi.org/10.1590/0104-07072014000600013

10. Brasil. Lei no 9.313 de 13 de novembro de 1996. Dispõe sobre a distribuição gratuita de medicamentos aos portadores do HIV e doentes de AIDS [Internet]. D.O.U., Brasília, DF, 14 nov 1996 [citado em 05 mar 2019]. Disponível em: http://www.planalto.gov.br/ccivil_03/leis/19313.htm 11. Ayres JRCM, Paiva V, França Junior I, Gravato N, Lacerda R, Della Negra M, et al. Vulnerability, human rights, and comprehensive health care needs of young people living with HIV/AIDS. Am J Public Health [Internet]. 2016 [citado em 05 mar 2019]; 96(6):1001-6. DOI: http://dx.doi.org/10.2105/AJPH.2004.060905

12. Ganong LH. Integrative reviews of nursing research. Res Nurs Health [Internet]. 1987 [citado em 05 mar 2019]; 10(1):1-11. DOI: https://doi.org/10.1002/nur.4770100103

13. Moher D, Liberati A, Tetzlaff J, Altman DG, The PRISMA Group. Preferred Reporting Items for Systematic Reviews and Meta-Analyses: the PRISMA statement. Plos Med. [Internet]. 2009 [citado em 05 mar 2019]; 6(7): e1000097. Disponível em:

https://www.ncbi.nlm.nih.gov/pmc/articles/PMC2707599/pdf/pmed.1000097.pdf. DOI: https://doi.org/10.1371/journal.pmed.1000097

14. Melnyk BM, Fineout-Overholt E, editors. Evidence-based practice in nursing \& healthcare: a guide to best practice. $2^{\text {nd }}$ ed Philadelphia: Wolters Kluwer/Lippincott; Williams \& Wilkins, 2011. 15. Muko KN, Tchanqwe GK, Nqwa VC, Njova L. Preventing mother-to-child transmission: factors affecting mothers' choice of feeding - a case study from Cameroon. SAHARA J. [Internet]. 2004 [citado em 05 mar 2019]; 1(3):132-8. DOI: http://dx.doi.org/10.1080/17290376.2004.9724836 16. Doherty T, Chopra M, Nkonki L, Jackson D, Persson LA. A Longitudinal qualitative study of infantfeeding decision making and practices among HIV-positive women in South Africa. J Nutr. [Internet]. 2006 [citado em 05 mar 2019]; 136(9):2421-6. DOI: http://dx.doi.org/10.1093/jn/136.9.2421

17. Doherty T, Chopra M, Nkonki L, Jackson D, Greiner T. Effect of the HIV epidemic on infant feeding in South Africa: "When they see me coming with the tins they laugh at me". Bull World Health Organ. [Internet]. 2006 [citado em 05 mar 2019]; 84(2):90-6. DOI: http://doi.org/10.2471/blt.04.019448 18. Machado MMT, Galvão MTG, Kerr-Pontes LRS, Cunha AJLA, Leite AJM, Lindsay AC, et al. Acesso e utilização de fórmula infantil e alimentos entre crianças nascidas de mulheres com HIV/AIDS. Rev 
Eletrônica Enferm. [Internet]. 2007 [citado em 05 mar 2019]; 9(3):669-711. Disponível em: https://www.fen.ufg.br/fen_revista/v9/n3/v9n3a10.htm

19. Réa MF, Santos RG, Sanchez-Moreno C. Quality of infant feeding counselling for HIV+ mothers in Brazil: challenges and achievements. Acta Paediatr. [Internet]. 2007 [citado em 05 mar 2019]; 96(1):94-9. DOI: http://dx.doi.org/10.1111/j.1651-2227.2007.00017.x

20. Chopra M, Rollins N. Infant feeding in the time of HIV: rapid assessment of infant feeding policy and programmes in four African countries scaling up prevention of mother to child transmission programmes. Arch Dis Child. [Internet]. 2008 [citado em 05 mar 2019]; 93(4):288-91. DOI: http://dx.doi.org/10.1136/adc.2006.096321

21. Buskens I, Jaffe A. Demotivating infant feeding counselling encounters in southern Africa: Do counsellors need more or different training? AIDS Care [Internet]. 2008 [citado em 05 mar 2019]; 20(3):337-45. DOI: http://dx.doi.org/10.1080/09540120701660346

22. Sibeko L, Coutsoudis A, Nzuza S, Gray-Donald K. Mothers' infant feeding experiences: constraints and supports for optimal feeding in an HIV-impacted urban community in South Africa. Publ Health Nutr. [Internet]. 2009 [citado em 05 mar 2019]; 12(11):1983-90. DOI: http://dx.doi.org/10.1017/S1368980009005199

23. Desclaux A, Alfeiri C. Counseling and choosing between infant-feeding options: overall limits and local interpretations by health care providers and women living with HIV in resource-poor countries (Burkina Faso, Cambodia, Cameroon). Soc Sci Med. [Internet]. 2009 [citado em 05 mar 2019]; 69(1):821-9. DOI: http://dx.doi.org/10.1016/j.socscimed.2009.06.007

24. Cames C, Saher A, Avassou KA, Cournil A, Meda N, Simondon KB. Acceptability and feasibility of infant-feeding options: experiences of HIV-infected mothers in the World Health Organization Kesho Bora mother-to-child transmission prevention (PMTCT) trial in Burkina Faso. Matern Child Nutr. [Internet]. 2010 [citado em 05 mar 2019]; 6(3):253-65. DOI: http://dx.doi.org/10.1111/j.1740-8709.2009.00201.x

25. Chisenga M, Siame J, Baisley K, Kasonka L, Filteau S. Determinants of infant feeding choices by Zambian mothers: a mixed quantitative and qualitative study. Matern Child Nutr. [Internet]. 2011 [citado em 05 mar 2019]; 7(2):148-59. DOI: http://dx.doi.org/10.1111/j.1740-8709.2010.00264.x 26. Ijumba P, Doherty T, Jackson D, Tomlinson M, Sanders D, Persson LA. Free formula milk in the prevention of mother-to-child transmission programme: voices of a peri-urban community in South Africa on policy change. Health Policy Plan. [Internet]. 2012 [citado em 05 mar 2019]; 28(7):761-8. DOI: http://dx.doi.org/10.1093/heapol/czs114

27. Nor B, Ahlberg BM, Doherty T, Zembe Y, Jackson D, Ekstrom EC. Mother's perceptions and experiences of infant feeding within a community-based peer counselling intervention in South Africa. Matern Child Nutr. [Internet]. 2012 [citado em 05 mar 2019]; 8(4):448-58. DOI: http://dx.doi.org/10.1186/s13006-017-0109-x

28. Zulliger R, Abrams EJ, Meyer L. Diversity of influences on infant feeding strategies in women living with HIV in Cape Town, South Africa: a mixed methods study. Trop Med Int Health [Internet]. 2013 [citado em 05 mar 2019]; 18(12):1547-54. DOI: http://dx.doi.org/10.1111/tmi.12212

29. Silva MR, Alvarenga WA, Dupas G. Experiência do cuidador no tratamento preventivo da criança exposta ao Vírus da Imunodeficiência Humana. Rev RENE (Online) [Internet]. 2014 [citado em 05 mar 2019]; 15(5):743-52. Disponível em: http://periodicos.ufc.br/rene/article/view/3232/2488 DOI: http://dx.doi.org/10.15253/2175-6783.2014000500003

30. Pacheco BP, Gomes GC, Xavier DM, Nobre CMG, Aquino DR. Dificuldades e facilidades da família para cuidar a criança com HIV/Aids. Esc Anna Nery Rev Enferm. [Internet]. 2016 [citado em 05 mar 2019]; 20(2):378-83. Disponível em: http://www.scielo.br/pdf/ean/v20n2/1414-8145-ean-2002-0378.pdf DOI: http://dx.doi.org/10.5935/1414-8145.20160052

31. Rensburg LJ, Nel R, Walsh CM. Knowledge, opinions and practices of healthcare workers related to infant feeding in the context of HIV. Health SA Gesondheid [Internet]. 2016 [citado em 05 mar 2019]; 21(1):129-36. DOI: http://dx.doi.org/10.1016/j.hsag.2015.12.001

32. Chaponda A, Goon DT, Hoque ME. Infant feeding practices among HIV-positive mothers at Tembisa hospital, South Africa. Afr J Prim Health Care Fam Med. [Internet]. 2017 [citado em 05 mar 2019]; 9(1):01278. DOI: http://dx.doi.org/10.4102/phcfm.v9i1.1278 
33. Suwankhong D, Liamputtong P. 'I was told not to do it but...': Infant feeding practices amongst HIV-positive women in southern Thailand. Midwifery [Internet]. 2017 [citado em 05 mar 2019]; 48:69-74. DOI: http://dx.doi.org/10.1016/j.midw.2017.03.007

34. Lang'at PC, Ogada I, Steenbeek A, MacDonald NE, Ochola S, Bor W, et al. Infant feeding practices among HIV-exposed infants less than 6 months of age in Bomet County, Kenya: an in-depth qualitative study of feeding choices. Arch Dis Child. [Internet]. 2018 [citado em 05 mar 2019]; 103(5):470-3. DOI: http://dx.doi.org/10.1136/archdischild-2017-314521

35. Nieuwoudt S, Manderson L. Frontline health workers and exclusive breastfeeding guidelines in an HIV endemic South African community: a qualitative exploration of policy translation. Int Breastfeed J. [Internet]. 2018 [citado em 25 set 2019]; 13:20. DOI: https://doi.org/10.1186/s13006-018-0164-y

36. Adeniyi OV, Ajayi AI, Issah M, Owolabi EO, Goon DT, Avramovic G, et al. Beyond health care providers' recommendations: understanding influences on infant feeding choices of women with HIV in the Eastern Cape, South Africa. Int Breastfeed J. [Internet]. 2019 [citado em 25 set 2019]; 14(7):1-12. DOI: http://dx.doi.org /10.1186/s13006-019-0201-5

\section{CONTRIBUIÇÕES}

Todas as autoras tiveram iguais contribuições no desenho, redação e, revisão do estudo.

\section{Como citar este artigo (Vancouver)}

Bick MA, Paula CC. Vulnerabilidade programática para insegurança alimentar de crianças expostas ao HIV: revisão integrativa. REFACS [Internet]. 2020 [citado em inserir dia, mês e ano de acesso]; 8(1):100-113. Disponível em: inserir link de acesso. DOI: inserir link do DOI.

\section{Como citar este artigo (ABNT)}

BICK, M. A.; PAULA, C. C. Vulnerabilidade programática para insegurança alimentar de crianças expostas ao HIV: revisão integrativa. REFACS, Uberaba, MG, v. 8, n. 1, p. 100-113, 2020. Disponível em: inserir link de acesso. Acesso em: inserir dia, mês e ano de acesso. DOI: inserir link do DOI.

\section{Como citar este artigo (APA)}

Bick, M.A. \& Paula, C.C. (2020). Vulnerabilidade programática para insegurança alimentar de crianças expostas ao HIV: revisão integrativa. REFACS, 8(1), 100-113. Recuperado em: inserir dia, mês e ano de acesso de inserir link de acesso. DOI: inserir link do DOI. 